\title{
A Coordinate-Based Approach for Exploiting Temporal-Spatial Diversity in Wireless Mesh Networks
}

\author{
Hyuk Lim \\ Department of Information and \\ Communications \\ Gwangju Institute of Science \\ and Technology \\ Gwangju, Republic of Korea \\ hlim@gist.ac.kr
}

\author{
Chaegwon Lim \\ School of Electrical \\ Engineering and Computer \\ Science \\ Seoul National University \\ Seoul, Republic of Korea \\ chaegwon@csl.snu.ac.kr
}

\author{
Jennifer C. Hou \\ Department of Computer \\ Science \\ University of Illinois at \\ Urbana-Champaign \\ Urbana, Illinois, USA \\ jhou@cs.uiuc.edu
}

\begin{abstract}
In this paper, we consider the problem of mitigating interference and improving network capacity in wireless mesh networks from the angle of temporal-spatial diversity. In a nutshell, while the achievable throughput on a multihop wireless path is limited by intra-flow interference, the overall capacity of a multihop wireless network can be increased by exploiting temporal-spatial diversity of concurrent transmissions that exist among a number of wireless links. Connections that are routed along multihop wireless paths can be scheduled to take place simultaneously if their transmissions do not interfere with each other (significantly).

To make a case of exploiting the temporal-spatial diversity to improve network capacity, we focus on transporting downstream traffic at gateway nodes with Internet access. We propose to construct, based on measurements of received signal strengths, a virtual coordinate system that is used to determine the sets of paths along which transmissions can take place with the least inter-flow interference. Based on the sets of non-interfering paths, the gateway node then determines the order with which a gateway node schedules frames of different connections to be transmitted. Through extensive simulation (with real-life measurement traces on an operational, city-wide wireless community network), we show that the downstream throughput of a gateway node in a wireless mesh network can be improved by $10-35 \%$ under a variety of network topologies and traffic distributions. This, coupled with the fact that the proposed approach requires only minor code change in the gateway nodes and does not require any additional hardware, makes it a viable option to improving network capacity in existing wireless mesh networks.
\end{abstract}

\footnotetext{
* The work reported in this paper was supported in part by NSF under Grant NSF ANI-02-21357 and MURI/ARO under a subcontract to Univ. California at Santa Cruz S0176939, and by the Ubiquitous Computing and Network (UCN) Project, the Ministry of Information and Communication (MIC) 21st Century Frontier R\&D Program in Korea.
}

Permission to make digital or hard copies of all or part of this work for personal or classroom use is granted without fee provided that copies are not made or distributed for profit or commercial advantage and that copies bear this notice and the full citation on the first page. To copy otherwise, to republish, to post on servers or to redistribute to lists, requires prior specific permission and/or a fee.

MobiCom'06, September 23-26, 2006, Los Angeles, California, USA.

Copyright 2006 ACM 1-59593-286-0/06/0009 ...\$5.00.

\section{Categories and Subject Descriptors}

C.2.1 [Network Architecture and Design]: Wireless communication

\section{General Terms \\ Algorithms, Performance}

\section{Keywords}

Wireless mesh network, Intra-/inter-flow interference, temporalspatial diversity, and topology discovery.

\section{INTRODUCTION}

Wireless mesh networks have emerged to be a new, cost-effective and performance-adaptive network paradigm for the next-generation wireless Internet. Targeting primarily for solving the well known last mile problem for broadband access [22,28], wireless mesh networks aim to offer high-speed coverage at a significantly lower deployment and maintenance cost. In such networks, most of the nodes are either stationary or less mobile. Only a fraction of nodes have direct access, and will serve as gateways, to the Internet. Several nodes serve as relays forwarding traffic from other nodes (as well as their own traffic) and maintain network-wide Internet connectivity, while the remaining nodes send packets along dynamically selected ad-hoc paths to gateway nodes with Internet access. Wireless mesh networks are preferable to existing cable/DSL based networks or wireless LANs (that provide WiFi access), due to the following potential advantages: (i) it is more cost effective, as service providers do not have to install a wired connection to each subscriber $(20-50 \mathrm{~K}$ per square mile to establish access, approximately $1 / 4$ of the cost incurred in high speed cable access); (ii) it is inherently more reliable, as each node has redundant paths to reach the Internet; (iii) the throughput attained by a user can be increased through routing via multiple, bandwidth-abundant paths (in contrast, in WLANs the shared bandwidth decreases as the number of users within a HotSpot increases); and (iv) the wireless network can readily extend their coverage by installing additional ad-hoc hops.

Several cities have planned (and/or partially deployed) wireless mesh networks, such as Bay Area Wireless User Group (BAWUG) [1], MIT Roofnet [3], Champaign-Urbana Community Wireless Network (CUWiN) [2], SFLan [5], Seattle Wireless [4], Southampton Open Wireless Network (SOWN) [6], and Wireless Leiden (in Netherlands) [3]. Although initial success has been reported in these efforts, a number of performance related problems have also been identified. Excessive packet losses/collision [8,11, 15], un- 
predictable channel behaviors $[8,11]$, inability to achieve throughput as afforded by IEEE 802.11 PHY/MAC, inability to find stable and high-throughput paths $[8,11]$, and lack of incentives to forward transit packets [21] are among those most cited to question whether or not the success of wireless mesh networks will sustain.

To deal with the problem of locating stable and high-throughput paths, several research efforts have been made to devise more sophisticated route metrics, e.g., expected transmission count (ETX) [12], round trip time (RTT) [7], and weighted cumulative expected transmission time (WCETT) [13]. Several of the aforementioned, throughput-related problems (e.g., excessive packet losses, unpredictable channel behaviors, and throughput degradation) have also been identified to be attributed, in part, by intra- and inter-flow interference $[10,15,32]$. Specifically, flows that are routed along different paths within the interference range compete for the channel bandwidth, resulting in inter-flow interference. On the other hand, consecutive packets in a single flow may be spread over the route to their destination and may interfere with one another, resulting in intra-flow interference. With the interference left uncontrolled, the operational range of a wireless mesh network would be limited to within a few hops, representing an insignificant stretch from the current wireless LANs or hotspots with respect to coverage.

To mitigate intra-/inter-flow interference, several complimentary approaches have been suggested (although they may not necessarily be proposed in the context of wireless mesh networks). For example, power control aims to enable each node to transmit with the minimal possible transmit power, subject to network connectivity [23-25]. With each node transmitting with the minimal possible power, the interference due to concurrent transmission is mitigated. Another (orthogonal) approach is to control the carrier sense threshold (with which a node determines whether the shared wireless medium is busy or idle) [34-36]. By having each node use a large carrier sense threshold, more concurrent connections can take place simultaneously (at the expense of decreased SINR and hence higher decoding failure rates). The third approach exploits the notion of channel diversity, and equips each node with one or more radios $[7,14,30,31]$. Concurrent transmission is made possible by having neighboring nodes transmit (and their corresponding receiver nodes receive) at different (non-overlapping) channels.

In this paper, we consider the problem of mitigating interference and improving network capacity in wireless mesh networks from the angle of temporal-spatial diversity. In a nutshell, while the achievable throughput on a multihop wireless path is limited by intra-flow interference, the overall capacity of a multihop wireless network can be increased by exploiting temporal-spatial diversity of concurrent transmissions that exist among a number of wireless links. Connections that are routed along multihop wireless paths can be scheduled to take place simultaneously if their transmissions do not interfere with each other (significantly). To make a case of exploiting the temporal-spatial diversity, we focus on transporting downstream traffic at gateway nodes with Internet access. This is because (i) most of the Internet accesses are intended for downloading large video/audio/text files; and (ii) by virtue of the way how wireless mesh networks operate, all the downloaded traffic is handled by gateway nodes. As a result, the downstream throughput at gateway nodes affects most significantly the performance as perceived by users.

There are two major issues that must be addressed in order to realize the notion of temporal-spatial diversity: (i) how to reason about the level of inter-flow interference among different paths; and (ii) how to schedule frame transmission among connections that potentially incur the least level of interference. To address the first issue, we propose to construct, based on the measurements of received signal strengths, a virtual coordinate system. This is in contrast to most existing work which relies on geographic locations of mesh nodes. With the use of the coordinate system, we are able to determine the sets of paths along which transmissions can take place with the least inter-flow interference. To address the second issue, we coordinate, based on the sets of non-interfering paths, the order with which a gateway node schedules transmission of frames of different connections. To allow a gateway node to send frames consecutively in an non-interruptible manner, we leverage the transmission opportunity (TXOP) option in the IEEE 802.11e specification [17]. That is, a gateway node that succeeds in grasping the medium is granted the right to use the medium for a period of time specified by TXOP. The gateway uses a TXOP to transmit multiple frames, with SIFS (instead of DIFS) as the interframe space between the sequence of DATA-ACK exchanges. If the DATA-ACK exchange has been completed, and there is still time remaining in the TXOP, the node may transmit another frame (after an idle time of SIFS), provided that the frame to be transmitted and its necessary acknowledgment can fit into the time remaining in the TXOP. The resulting scheme is termed as coordinated transmission (CoTx).

To evaluate the performance of the proposed coordinate system and CoTx, we have made real-life RSS measurements on the Champaign - Urbana Community Wireless Network (CUWiN). Note that CUWiN is one of the first fully-operational, community-owned wireless mesh networks with self-configuring software. The latest software release that includes scalability improvement has been made in January 2006. At the time of writing the manuscript, a total of 30 wireless nodes have been set up and operational in the downtown area. Through extensive simulation with these measurement traces, we show that (1) the virtual coordinate system constructed with the received signal strength infers interference in a more accurate manner than the geographical locations of nodes (as was used in most existing work); and (2) with the proposed approach, the downstream throughput of a gateway node in a wireless mesh network can be improved by $10-35 \%$ in a variety of network topologies and traffic distributions. This, coupled with the fact that the proposed approach needs only minor code change in the gateway nodes and does not require additional hardware, makes it a viable option to improving network capacity in existing wireless mesh networks.

The reminder of the paper is organized as follows. In Section 2, we give a succinct summary of IEEE 802.11 and its associated intra-/inter-flow interference problems. We also motivate our proposed work with an illustrative example there. In Section 3, we elaborate on how gateway nodes collect necessary information of received signal strengths and construct the coordinate system. In Section 4, we discuss the procedures with which gateway nodes coordinate the order of frame transmission among different connections. We also present an implementation with IEEE 802.11e. This is then followed by a discussion of related work in Section 5 and the performance evaluation (with real-life measurement traces) in Section 6. Finally, we conclude the paper in Section 7 with a list of future research agendas.

\section{MOTIVATION}

We consider a stationary, multihop wireless network that operates on IEEE 802.11 distributed coordination function (DCF). The basic access method of DCF is carrier sensing multiple access with collision avoidance (CSMA/CA). A node that intends to transmit senses the channel and defers its transmission while the channel is sensed busy. In addition to the basic method, the request-tosend/clear-to-send (RTS/CTS) mechanism can be optionally used, in the hope to solve the hidden/exposed terminal problems [9]. It 


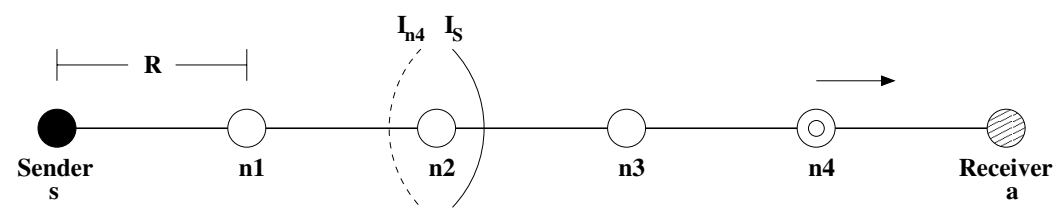

(a) A single multihop path.

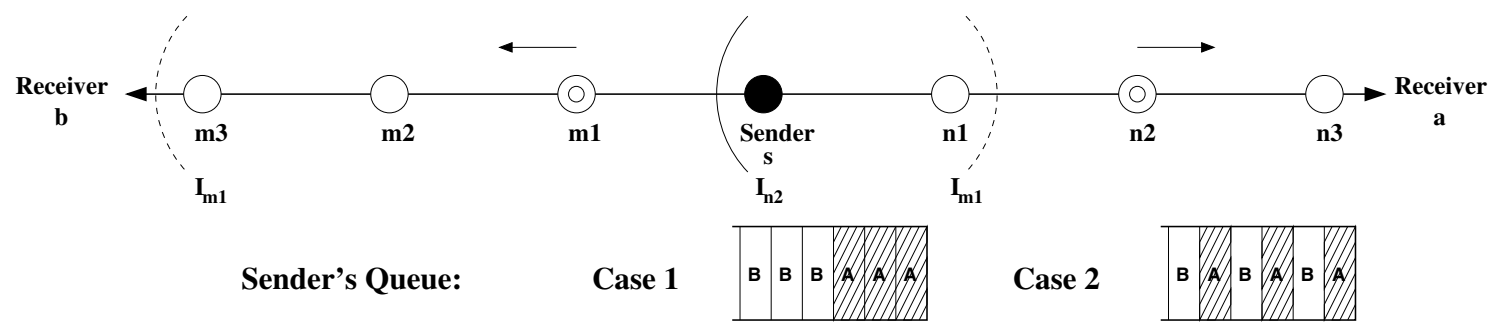

(b) Two multihop paths in the opposite directions.

Figure 1: Illustration of temporal-spatial diversity in multihop wireless networks.

has been shown in the literature that the RTS/CTS floor acquisition mechanism handles the hidden/exposed sender problem well, but fails to solve the hidden/exposed receiver problem [9, 15,33]. As a result, the RTS/CTS mechanism is often turned off in practice for the sake of improving the throughput performance.

Figure 1 illustrates how the interference (caused by the hidden/ exposed terminals) affects the network throughput in multiple-hop wireless network. In Figure 1, adjacent nodes are within the transmission range of each other, and the interference range is approximately twice of the transmission range. Note that we define the interference range as the distance to the sender within which the signal-to-interference ratio (SIR) is greater than a certain threshold called the SIR threshold. Let $R$ and $I_{\mathrm{x}}$ denote, respectively, the transmission range and the set of nodes within the interference range of node $\mathbf{x}$. For example, $I_{\mathbf{s}}=\{\mathbf{s}, \mathbf{n 1}, \mathbf{n 2}\}$ in Figure 1. As shown in Figure 1(a), sender $\mathbf{s}$ transmits a sequence of data frames to receiver a. Due to intra-flow interference, the number of concurrent transmissions that can take place without interfering with one another is limited. For example, when $\mathbf{s}$ is transmitting frames to $\mathbf{n} 1$ (denoted by $\mathbf{s} \longmapsto \mathbf{n} 1$ ), only $\mathbf{n} 4$ can transmit simultaneously without interfering with the transmission of $\mathbf{s} \longmapsto \mathbf{n 1}$. This restriction on the number of concurrent transmissions makes it difficult for a connection to attain high throughput along a single multihop path.

In a large network consisting of more than one multiple-hop paths, it is, however, possible to increase the number of concurrent transmissions by exploiting temporal-spatial diversity. Figure 1(b) shows a simple wireless network with two connections ( $\mathbf{s} \rightarrow \mathbf{a}$ and $\mathbf{s} \rightarrow \mathbf{b}$ ) along two multihop paths in opposite directions. Now we consider two extreme cases at $\mathbf{s}$ : i) the frames destined for a are transmitted first, followed by those destined for $\mathbf{b}$ and ii) the frames destined for $\mathbf{a}$ interleave with those destined for $\mathbf{b}$. In case (i), spatial diversity that exists along the two different paths cannot be exploited, and the number of concurrent transmissions is almost the same as that in Figure 1(a). This is because after the first frame of connection $\mathbf{s} \rightarrow \mathbf{a}$ is transmitted by $\mathbf{s}$, it will complete with subsequent frames from $\mathbf{s}$ until it is at least three hops away from any subsequent frame (e.g., it reaches $\mathbf{n}_{4}$ while the second frame of $\mathbf{s} \rightarrow \mathbf{a}$ is still at $\mathbf{s})$.

On the other hand, in case (ii) of Figure 1(b), as the frames destined for $\mathbf{a}$ interleave with those destined for $\mathbf{b}$, it is possible for concurrent transmissions to take place without interfering with each other. For example, once the first frames destined for $\mathbf{a}$ and $\mathbf{b}$ reach, respectively, $\mathbf{n}_{2}$ and $\mathbf{m}_{\mathbf{1}}$, the transmission $\mathbf{n}_{\mathbf{2}} \longmapsto \mathbf{n}_{\mathbf{3}}$ can take place concurrently with $\mathbf{m}_{\mathbf{1}} \longmapsto \mathbf{m}_{\mathbf{2}}$. As a matter of fact, if $\mathbf{m}_{\mathbf{1}}$ and $\mathbf{n}_{\mathbf{1}}$ initiates the transmission at approximately the same time (and the RTS/CTS mechanism is not exercised), the transmissions $\mathbf{m}_{\mathbf{1}} \longmapsto \mathbf{m}_{\mathbf{2}}$ and $\mathbf{n}_{\mathbf{1}} \longmapsto \mathbf{n}_{\mathbf{2}}$ may simultaneously take place.

As shown in the above example, intra-/inter-flow interference is dependent both upon how traffic is distributed and how traffic is routed along potentially interfering paths. As a result, to explore temporal-spatial diversity, we have to consider the following two issues: (i) how to reason about the level of inter-flow interference among different paths, and find sets of paths along which frames can be transmitted concurrently with the least inter-flow interference; and (ii) how to schedule frame transmission among connections that are identified to the least inter-flow transmission. To address the first issue, we will construct in Section 3, based on measurements of received signal strengths, a virtual coordinate system. Then we elaborate in Section 4 on how we schedule transmission of frames of different connections.

\section{DETERMINING VIRTUAL COORDINATES BASED ON SIGNAL STRENGTHS}

To make a case of exploiting temporal-spatial diversity to improve network capacity, we focus on transporting downstream traffic at gateway nodes with Internet access. This is because most of the gateway nodes are responsible for transporting a large amount of downstream traffic, and how they schedule transmission of frames to downstream mesh nodes will have a significant impact on the performance as perceived by users.

To infer the level of inter-flow interference among different paths, one may choose to use geographic locations of (next-hop) nodes as the references. Although this information can be readily obtained by GPS or as part of the static network configuration, it is sometimes quite misleading. For example, even though two next-hop nodes are geographically close to each other, the interference may not be significant if there is an obstacle between them. Due to this reason, we propose to exploit received signal strength (RSS) measurements among neighbors (to be defined below) as the references. This is because RSS measurements are more "representative" in determining the level of interferences between nodes. Moreover, they can be readily obtained through the sensory functions which are implemented in most of the modern IEEE 802.11 interfaces. Based 
on the RSS measurements among neighbors, we will construct a virtual coordinate system and use the "virtual distance" between mesh nodes to infer the level of interferences between them [20].

\subsection{Measuring RSSs Between Mesh Nodes}

To find the sets of paths with the least inter-flow interference, we will construct, for each gateway node $(\mathrm{GN})$, a virtual coordinate system centered at the GN. The first step to constructing such a virtual coordinate system is to instrument nodes that can communicate with the GN directly or through a "relay" node in between to perform RSS measurements. Each such node periodically transmits hello packets, measures RSSs from the other nodes, and reports the measurements to its neighbors. In some sense, the RSS measurements are performed within two hops of the GN. If we increase the measurement area to within $h$ hops from the $\mathrm{GN}(h>2)$, it is possible to infer the level interference between nodes that are far from the GN and find the sets of paths with the least interferences more accurately. However, this is at the expense of higher control overhead and higher complexity of the algorithm that selects the set of interference-free paths. For practicality and scalability, we restrict the measurement area to be within two hops from from the GN.

Through exchange of hello packets, a GN gathers the RSS measurement between itself and a node $\mathbf{m}$ that can directly communicate with it and that between a neighbor node of $\mathbf{m}$ 's and $\mathbf{m}$. Let $\mathcal{M}(n)$ denote the set of neighbor nodes that can directly communicate with $\mathbf{n}$ and $\mathbf{n}$ itself. Then, the RSS measurements between $\mathbf{n}$ and nodes in $\mathcal{M}(n)$ can be written in an $p \times p$ square matrix $\mathbf{S}=\left[s_{i j}\right]$ for $i, j \in\{1, \cdots, p\}$, where $s_{i j}$ is the (- RSS) ${ }^{1}$ measurement made in $\mathrm{dBm}$ by the $i$ th node to $j$ th node, $s_{i i}=0$, and $p=|\mathcal{M}(n)|$ is the number of $\mathbf{n}$ 's neighbor nodes plus 1 . $\mathbf{S}$ is termed as the signal strength matrix, and will be used to construct a virtual coordinate system centered around $\mathbf{n}$.

One point that is worthy of mentioning is that all the components of $\mathbf{S}$ may not be available because the distances between some neighbor nodes may be occasionally larger than the wireless transmission range. For example, two nodes $\ell$ and $\mathbf{m}$ are the neighbor nodes of the GN, but each node may be located outside the transmission range of the other node. We will further discuss in Section 3.2 how we deal with the case that some of the pairwise RSS measurements are not available.

After the coordinates of nodes in $\mathcal{M}(n)$ are obtained, one can further derive the coordinate of a node $\mathbf{k}$ that cannot directly communicate with $\mathbf{n}$ but can via relay node(s) in $\mathcal{M}(n)$, with the use of RSS measurements made between $\mathbf{k}$ and relay nodes that can directly communicate with node $\mathbf{n}$. In some sense, construction of the virtual coordinate system proceeds in a ring-by-ring manner, starting from the innermost ring composed of $\mathbf{n}$ 's neighbor nodes and proceeding outward.

\subsection{Constructing Virtual Coordinate System}

The RSS measurements between $\mathbf{n}$ and its neighbors are represented by the $p \times p$ square matrix $\mathbf{S}$, the columns of which can be considered as the coordinates of the corresponding nodes in a $p$ dimension space. Note that the $i$ th column vector of $\mathbf{S}$ is the RSSs measured by the $i$ th node from all the nodes in $\mathcal{M}(G N)$. As these coordinates are correlated with each other, it is difficult to identify components that play an important role in determining the interferences. Hence we propose to construct an orthogonal coordinate system with a smaller dimensionality by using singular value decomposition (SVD). First, the signal strength matrix $\mathbf{S}$ is shifted to

\footnotetext{
${ }^{1}$ As the sign of the RSS measurement is negated, a smaller value of $s_{i j}$ implies stronger signal strength.
}

its center by subtracting the mean vector of $\mathbf{S}$. Second, the shifted matrix $\overline{\mathbf{S}}$ is decomposed by SVD as follows:

$$
\overline{\mathbf{S}}=\mathbf{U} \cdot \mathbf{W} \cdot \mathbf{V}^{T},
$$

where $\mathbf{U}$ and $\mathbf{V}$ are column and row orthogonal matrices, and $\mathbf{W}$ is the diagonal matrix with the singular values of $\overline{\mathbf{S}}$ in the decreasing order [27]. The columns of the $p \times p$ matrix $\mathbf{U}=\left[\mathbf{u}_{1}, \ldots, \mathbf{u}_{p}\right]$ are the orthogonal basis of the new subspace. Last, by using the first $q$ columns of $\mathbf{U}$ denoted by $\mathbf{U}_{q}$, we project the $p$-dimensional space into a new $q$-dimensional space:

$$
\mathbf{c}_{i}=\mathbf{U}_{q}^{T} \cdot \overline{\mathbf{s}}_{i}=\left[\mathbf{u}_{1}, \ldots, \mathbf{u}_{q}\right]^{T} \cdot \overline{\mathbf{s}}_{i},
$$

where $\mathbf{c}_{i}$ is the new coordinate of the $i$ th node, and $\overline{\mathbf{s}}_{i}$ is the $i$ th column vector of $\overline{\mathbf{S}}$.

After obtaining the coordinates, we have to perform two postprocessing operations. The first operation is to translate the coordinate system so that the GN (i.e., $\mathbf{c}_{G N}$ ) becomes the origin. The second operation is to scale the coordinate system so that the distance between two nodes coincides with the corresponding, actual measured signal strength. The optimal scaling factor $\alpha^{*}$ that minimizes the discrepancy between the Euclidean distance and the measured signal strength can be determined by minimizing the following objective function

$$
J_{1}(\alpha)=\sum_{i}^{p} \sum_{j}^{p}\left(L\left(\alpha \mathbf{c}_{i}, \alpha \mathbf{c}_{j}\right)-s_{i j}\right)^{2},
$$

where the $L$ is the Euclidean distance between two vectors (i.e., $\left.L(\mathbf{x}, \mathbf{y})=\sqrt{(\mathbf{x}-\mathbf{y})^{T}(\mathbf{x}-\mathbf{y})}\right)$. After a few algebraic operations, the positive solution, $\alpha^{*}$, can be shown to be

$$
\alpha^{*}=\frac{\sum_{i}^{p} \sum_{j}^{p} s_{i j} L\left(\mathbf{c}_{i}, \mathbf{c}_{j}\right)}{\sum_{i}^{p} \sum_{j}^{p} L\left(\mathbf{c}_{i}, \mathbf{c}_{j}\right)^{2}} .
$$

The new coordinate of a node is written by

$$
\mathbf{x}_{i}=\alpha^{*}\left(\mathbf{c}_{i}-\mathbf{c}_{G N}\right) .
$$

Determining Coordinates for Nodes That Are Two Hops Away. Recall that as shown in the illustrative example in Section 2 , if two nodes are outside the transmission range but within the interference range of each other, their transmissions interfere with each other, but cannot be directly measured by either node without the support of intermediate nodes. As a result, the GN has to be aware of the level of interference at its two-hop away nodes in order to exploit spatial diversity (Fig. 1 (b)). That is, the virtual coordinate system has to be "extended" to include the coordinates of nodes that are two hops away.

As discussed in Section 3.1, a neighbor node of the GN measures RSSs from its neighbor nodes. For a node $\mathbf{k}$ that is two hops from $\mathrm{GN}$, if $l=|\mathcal{M}(\mathrm{GN}) \cap \mathcal{M}(\mathbf{k})| \geq q+1$, we obtain the coordinate of $\mathbf{k}, \mathbf{x}_{k}$, by minimizing the following objective function:

$$
J_{2}\left(\mathbf{x}_{k}\right)=\sum_{i \in \mathcal{M}(\mathrm{GN}) \cap \mathcal{M}(\mathbf{k})}\left(L\left(\mathbf{x}_{i}, \mathbf{x}_{k}\right)-s_{i k}\right)^{2} .
$$

Because solving this non-linear optimization problem incurs high computational complexity, we use an alternative, non-iterative multilateration algorithm [19]: From the quadratic version of the Euclidean distance equations (i.e., $L\left(\mathbf{x}_{k}, \mathbf{x}_{i}\right)^{2}=\sum_{j=1}^{q}\left(x_{k}^{j}-x_{i}^{j}\right)^{2}=$ $s_{i k}^{2}$ and $x^{j}$ is the $j$ th component of $\mathbf{x}$.), a linear system is derived by subtracting one of the equations from the other equations.

$$
\mathbf{A x}_{\mathbf{k}}=\mathbf{b}
$$




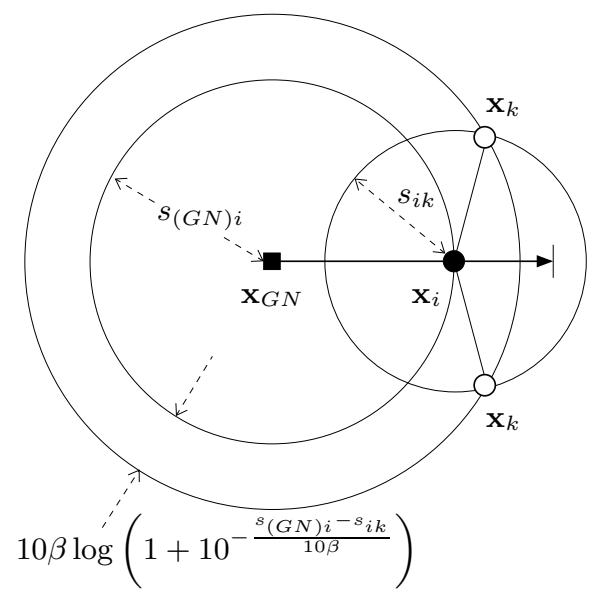

Figure 2: How to determine the coordinate of a node that is two hops away from a GN when the number of RSS measurements is not sufficient.

and

$$
\mathbf{A}=2\left[\begin{array}{c}
\left(\mathbf{x}_{1}-\mathbf{x}_{l}\right)^{T} \\
\vdots \\
\left(\mathbf{x}_{l-1}-\mathbf{x}_{l}\right)^{T}
\end{array}\right], \mathbf{b}=\left[\begin{array}{c}
\mathbf{x}_{1}^{T} \mathbf{x}_{1}-s_{1 k}^{2} \\
\vdots \\
\mathbf{x}_{l-1}^{T} \mathbf{x}_{l-1}-s_{(l-1) k}^{2}
\end{array}\right]-\eta \mathbb{1}_{l-1},
$$

where $\eta=\left(\mathbf{x}_{l}^{T} \mathbf{x}_{l}-s_{l k}^{2}\right)$ and $\mathbb{1}_{M-1} \in \mathbb{R}^{M-1}$ is the 1's column vector. The coordinate is given by the least square solution of the linear system, i.e.,

$$
\mathbf{x}_{k}=\left(\mathbf{A}^{T} \mathbf{A}\right)^{-1} \mathbf{A}^{T} \mathbf{b} .
$$

It requires neither a judicious guess of the initial location nor a time-consuming iteration process to solve Eq. (4).

In the case that node $\mathbf{k}$ does not have sufficient neighbor nodes (i.e., $l<q+1$ ), it is not possible to uniquely determine the coordinate of $\mathbf{k}$. In this case, we obtain the coordinate by making the assumption that the signal strength is inversely proportional to $d^{\beta}$, where $d$ is the geographical distance between the nodes and $\beta$ is the path loss coefficient. Figure 2 illustrates an example in which the dimension of the coordinate system is two, and only two RSS measurements $s_{(G N) i}$ and $s_{i k}$ are available. From the inequality for the geographic distances between the nodes (i.e., $\left.d_{(G N) k} \leq d_{(G N) i}+d_{i k}\right)$, the RSS between GN and $\mathbf{k}$ is bounded under the assumption as

$$
s_{(G N) k} \leq s_{(G N) i}+10 \beta \log \left(1+10^{-\frac{s_{(G N) i}-s_{i k}}{10 \beta}}\right) .
$$

By taking the bound, we have two possible coordinates for $\mathbf{k}$, labeled as $\mathbf{x}_{k}$ in Figure 2, which are symmetric with respect to the corresponding one-hop neighbor, $\mathbf{x}_{i}$, of the GN. Since the symmetry holds in general, we simply take a vector whose direction is the same as $\mathbf{x}_{i}$ and whose magnitude is the average of $s_{i k}$ and the bound in Eq. (6) for $\mathbf{x}_{k}$. That is,

$$
\mathbf{x}_{k}=\left(1+\frac{s_{i k}+10 \beta \log \left(1+10^{\left.-\frac{s_{(G N) i-s_{i k}}^{10 \beta}}{10 \beta}\right)}\right.}{2\left|\mathbf{x}_{i}\right|}\right) \mathbf{x}_{i} .
$$

Note that this approach renders an approximate estimate of $x_{k}$ and errors on the pessimistic side when there exist obstacles between the GN and node $\mathbf{k}$.

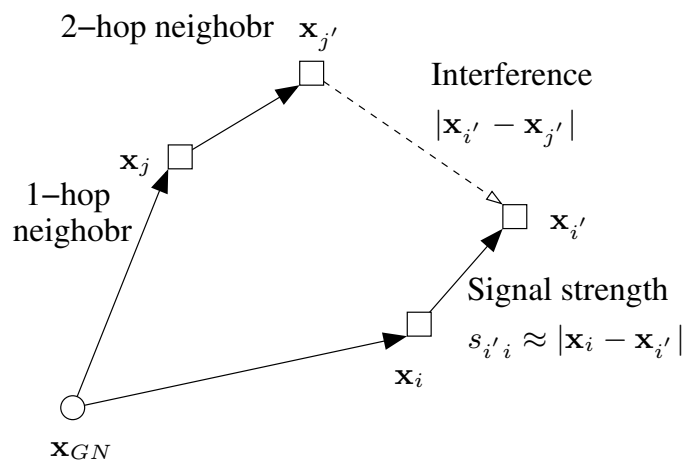

Figure 3: How to infer interference between nodes that are 2 hops away from a gateway node with the use of their coordinates.

\subsection{Identifying Paths with Least Interference}

We can infer the interferences between mesh nodes by computing the Euclidean distances in the coordinate system. If the distance between two nodes is large, it implies that they will not interfere with each other's transmission. In this subsection, we devise an algorithm that selects, based on the signal strength prediction between nodes in the coordinate system, paths along which frames can be transmitted concurrently with the least inter-flow interference.

Recall that as shown in Figure 1(b), it is not possible to avoid inter-flow interference near $\mathbf{s}$ because $\mathbf{s}, \mathbf{m} \mathbf{1}, \mathbf{n} \mathbf{1}$ are so close to each other. However, if the interference range is $\tau$ times larger than the transmission range and $2<\tau \leq 3$, then both $\mathbf{m} \mathbf{1}$ and $\mathbf{n 2}$ (or $\mathbf{m} \mathbf{2}$ and $\mathbf{n 1}$ ) can transmit at the same time. Motivated by this example, we take into account of the first two hops of paths in determining the set of connections whose frames can be transmitted concurrently. In some sense, we determine the set of first relay nodes that give the least interference when frames are being forwarded by them.

Figure 3 illustrates how to inter the interference between nodes that are two hops away from the GN with the use of their coordinates. In the figure, the coordinates of the 1-hop neighbor nodes of a GN are labeled as $\mathbf{x}_{i}$ and $\mathbf{x}_{j}$ and calculated in Eq. (3), while those of the corresponding two-hop neighbor nodes are labeled as $\mathbf{x}_{i^{\prime}}$ and $\mathbf{x}_{j^{\prime}}$ and calculated in Eqs. (5) or (7). The signal-to-interference ratio (SIR) at $\mathbf{x}_{i^{\prime}}$ due to the interference from either $\mathbf{x}_{j}$ or $\mathbf{x}_{j^{\prime}}$, denoted by $\operatorname{SIR}_{i^{\prime}}\left(j^{\prime}\right)$, is given by

$$
\operatorname{SIR}_{i^{\prime}}\left(j^{\prime}\right)=\min \left(\left|\mathbf{x}_{i^{\prime}}-\mathbf{x}_{j}\right|,\left|\mathbf{x}_{i^{\prime}}-\mathbf{x}_{j^{\prime}}\right|\right)-s_{i^{\prime} i} .
$$

For each pair of nodes, if SIR is larger than an SIR threshold $\gamma$ (in $\mathrm{dB})$, we consider the pair as one with negligible inter-flow interference. We construct an interference table $T$, of which the $i j$ th entry is given by

$$
t_{i j}=\left\{\begin{array}{cc}
1 & \text { if } \operatorname{SIR}_{i}(j)>\gamma \\
0 & \text { otherwise }
\end{array}\right.
$$

We refer to the table $T$ to determine whether the transmission of a node will interfere with another. It is important to note that the probing process is locally performed among neighbor nodes of a GN and the process of constructing the interference table is only performed at the GN.

One issue that is worthy of discussion is the availability of routing information. To infer the potential interference between transmissions at two nodes that are two hops away from the GN (Eq. (9)), 


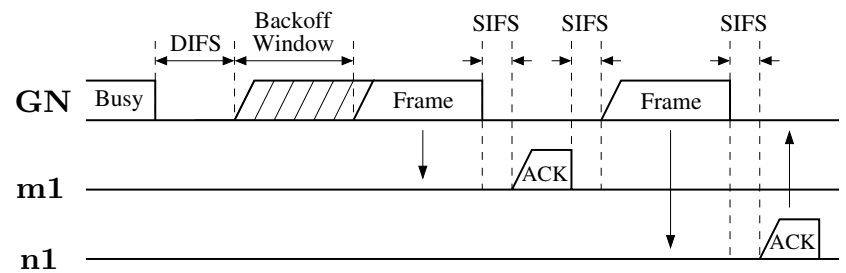

Figure 4: A scenario in which the GN coordinates transmission between itself and its neighbor nodes $\mathrm{m} 1$ and $\mathrm{n} 1$ in Fig. 1 .

it is required that the routing information up to two hops be available. While the next node $\mathbf{m}$ to which a frame is to be transmitted is usually available at GN, whether or not the next node of $\mathbf{m}$ is available to the GN depends upon the selection of the routing algorithm. For example, under the dynamic source routing (DSR) algorithm, the GN maintains the route cache for source routing and can obtain the two-hop node information without any overhead. In the case that the two-hop node information is not available at the GN, the information can be piggy-backed as part of the hello packets.

\section{COORDINATING ORDER OF TRANSMISSION}

To enable the GN to coordinate the order of transmission and fully explore temporal-spatial diversity, we propose a coordinated transmission (CoTx) scheme. There are two major operations of CoTx: first the order of frame transmissions should be determine, with the objective of fully exploiting temporal-spatial diversity. Second, the GN should be given the chance to transmit a sequence of frames, once it grasps the medium. For example, in Figure 1 (b) the best way to increase the level of temporal-spatial diversity is to enable node $\mathbf{s}$ to transmit frames alternatively to each receiver. If after the GN transmits a frame to $\mathbf{n} \mathbf{1}$ it cannot continue holding the medium and transmit a frame to $\mathbf{m} \mathbf{1}$ until the first frame reaches $\mathbf{n} 3$, then the number of concurrent transmissions essentially does not increase. Figure 4 shows one possible scenario of how the GN coordinates the transmission between itself and its neighbor nodes $\mathbf{m} 1$ and $\mathbf{n} 1$. After the channel is sensed free for a DIFS time interval the GN waits for a random backoff interval and transmits the first frame to $\mathbf{m} \mathbf{1}$. After that, the GN chooses a frame that does not interfere with transmission of the first frame (in the near future) and transmits it without backoff.

\subsection{Determining the Transmission Order}

To select subsequent frames that give the least interference, we leverage the interference table $T$ computed in Section 3.3. Specifically, after sending the first frame, the GN looks up a candidate frame from the head of the queue in the logical link control (LLC) layer. Let $\Lambda$ denote the set of neighbor nodes to which frames were sent after the GN grasps the medium. For example, $\Lambda=\{\mathbf{m} 1\}$ after the first frame is transmitted in Figure 4. The GN looks up to $N$ frames in the LLC queue in order to locate a frame $f$ that satisfies $t_{\text {routing }(f) i}=1$ for $\forall i \in \Lambda$, where routing $(\cdot)$ is the function that returns the next hop of a frame.

\subsection{Implementing CoTx on top of IEEE 802.11}

CoTx requires that a GN be granted an extended interval (sufficient to transmit up to $N$ frames) after it grasps the medium. This can be realized with the functions available in IEEE 802.11e enhanced distributed channel access (EDCA) draft [17]. In what fol- lows, we first give a succinct overview of EDCA and then discuss how we will leverage the functions provided in EDCA to implement CoTx.

IEEE 802.11e EDCA has been proposed to support quality-ofservice (QoS) in WLANs. In EDCA, several parameters control how and when a node gains access to the medium among different priority levels (called access categories (ACs)), so as to favor/disfavor data transmission from high-priority/low-priority flows. These parameters include the minimum idle delay before contention (AIFS), the minimum and maximum contention windows $\left(C W_{\min }\right.$ and $C W_{\max }$ ), and the transmission opportunity limit (TXOP). All the parameters can be dynamically updated by the access point (AP) through the EDCA parameter set, and are sent from the AP as part of the beacon frames and probe/re-association response frames. This adjustment allows stations in the WLAN to adapt to changing conditions, and gives the AP the ability to manage the overall QoS performance.

The function with which we will leverage to enable the GN to transmit a sequence of $N$ frames is TXOP. Specifically, a station that succeeds in grasping the medium is granted a transmission opportunity (TXOP) - the right to use the medium and transmit multiple frames without backoff. If the DATA-ACK exchange sequence has been completed, and there is still time remaining in the TXOP, the station may transmit another frame in the same access category, provided that the frame to be transmitted and its necessary acknowledgment can fit into the time remaining in the TXOP. In CoTx, we enable the GN to set its TXOP to an interval sufficient to transmit $N$ frames. In this manner, a GN can hold the medium and transmit up to $N$ frames consecutively.

When the GN finishes transmitting the last frame eligible for transmission based on $T$, it relinquishes the medium and the neighbor nodes will complete for the medium to relay frames that were sent to them by the GN. In order to give more opportunities to its neighbors, the GN is instrumented to set a contention window size that is larger than that originally specified in IEEE 802.11 DCF. Specifically, the contention window is set to the sum of the backoff intervals that were skipped when the GN is in possession of the medium. That is, the contention window is set to

$$
C W=\min \left(|\Lambda| \times C W_{\min }, C W_{\max }\right),
$$

where $C W, C W_{\min }$, and $C W_{\max }$ are the current, maximum, and the minimum contention window sizes, respectively. In the case that the every frame in the queue in the LLC layer interferes with those transmitted earlier, only one frame is transmitted (i.e., $|\Lambda|=$ 1 ), and $C W$ in Eq. (10) essentially falls back to that originally specified in IEEE 802.11. We can leverage the function of setting $C W_{\min }$ to realize the implementation. That is, $\mathrm{GN}$ sets its $C W_{\min }$ value to the value specified in Eq. (10) before the contention period starts, and sets it back to the previous value after the contention period finishes.

\subsection{Algorithms for Coordinated Transmission in LLC/MAC Layers}

The pseudo-code of the proposed algorithm is given in Algorithms 1,2, and 3. They should be, respectively, implemented in the LLC layer (Algorithm 1) and the MAC layer (Algorithm 2) at a GN.

Algorithm 1 outlines how the LLC layer operates to support the coordinated transmission in the MAC layer. The LLC layer selects, based on $\Lambda$ and $T$ (computed in Section 3.3), a frame whose transmission will not interfere with the frames sent earlier in this run of coordinated transmission. The LLC layer inspects in sequence $N$ frames from the head of the queue. If the queue is not empty and an 

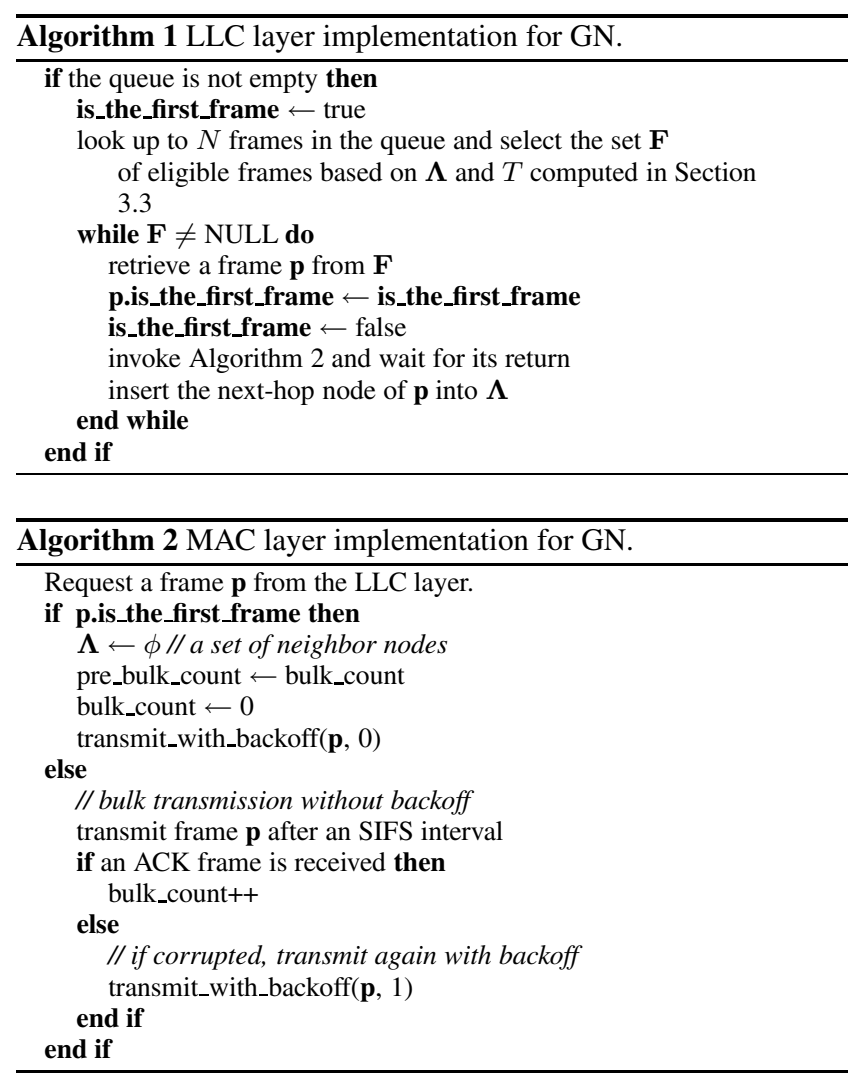

appropriate frame is identified, the LLC layer updates $\Lambda$ and passes the frame onto the MAC layer.

Algorithms 2 and 3 show how the MAC layer implements the proposed algorithm. Algorithm 2 is called whenever a frame arrives from the LLC layer. If the frame is the first for this run of coordinated transmission, the transmit_with_backoff subroutine in Algorithm 3 is called to transmit the frame after a random backoff interval. The backoff value is randomly drawn from the range of $[0, C W-1]$ and $C W$ is computed by Eq. (10).

When a GN detects collision in the middle of coordinated transmission, it follows the binary exponential backoff algorithm defined in IEEE 802.11 DCF (Algorithm 3). Even in this case, the GN does not terminate its bulk transmission but continues with an additional random backoff interval to avoid potential collisions. If a frame fails to be transmitted for retransmission_limit times, it is dropped but the GN continues with its bulk transmission and attempts to transmit the next frame after an SIFS interval.

\section{RELATED WORK}

Several approaches have been proposed to control the "sharing range" of a wireless link and ultimately the degree of spatial reuse: the transmit power each node uses, the carrier sense threshold each node uses to determine if the shared medium is idle, and the channel on which a node transmits.

Transmission power control: Transmission power control (TPC) is an approach that enables each node to transmit with the minimal possible transmit power in order to decrease the interference between adjacent nodes and to improve the spatial reuse [23-25]. In [23], Monks et al. proposed a power control protocol called PCMA, in which the receiver advertises its interference margin that it can tolerate on an out-of-band channel and the trans-

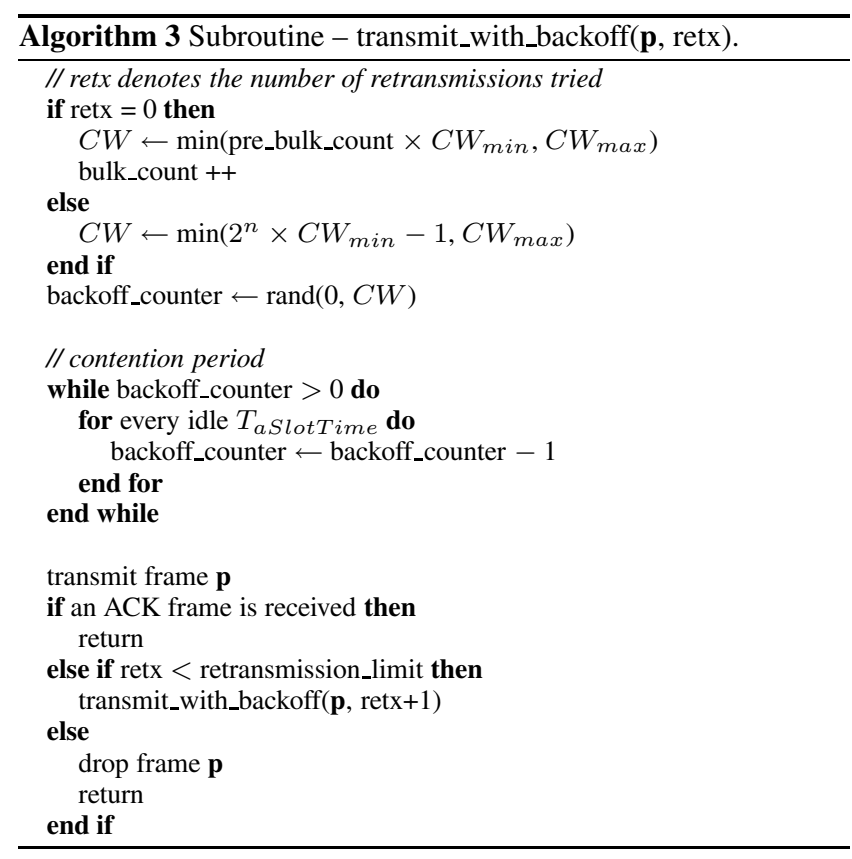

mitter selects its transmit power that does not disrupt any ongoing transmissions. Muqattash and Krunz proposed similar power control protocols called PCDC and POWMAC in [24,25] respectively. The PCDC protocol constructs the network topology by overhearing RTS and CTS packets, and the computed interference margin is announced on an out-of-band channel. The POWMAC protocol, on the other hand, uses a single channel for exchanging the interference margin information.

Carrier sense threshold tuning:. The carrier sense threshold (with which a node determines whether the shared wireless medium is busy or idle) can be adjusted to improve the spatial reuse. Zhu et al. $[35,36]$ showed that the physical carrier sensing with an tunable threshold can significantly enhance the overall network throughput by enabling simultaneous transmission while maintaining the minimal separation distance for spatial reuse. Yang and Vaidya [34] considered several factors such as MAC overhead, transmission rate, and network density in selecting optimal carrier sense threshold that maximize the aggregate throughput.

Channel diversity:. Several network architectures have been designed and evaluated to enable concurrent transmission by equipping nodes with multiple radios and having neighboring nodes transmit at different (non-overlapping) channels [14,31]. While the interference between different channels is negligible in such networks, routing and channel assignment issues have to be appropriately considered in order for a sender-receiver pair to tune to the same channel. Raman and Chebrolu [30] envision a mesh network which each node is equipped with multiple radios with directional antenna and wireless links are long-distance and point-topoint. These multiple radios are synchronized so that they operate in either the transmitting or the receiving mode without interfering with each other.

Intra-flow interference mitigation:. Fu et al. [15] investigated the effect of intra-flow interference that exist on a single multiple-hop path on the TCP performance. They observed that an optimized TCP window size mitigates intra-flow interference and renders higher throughput intra-flow interference. Based on the 


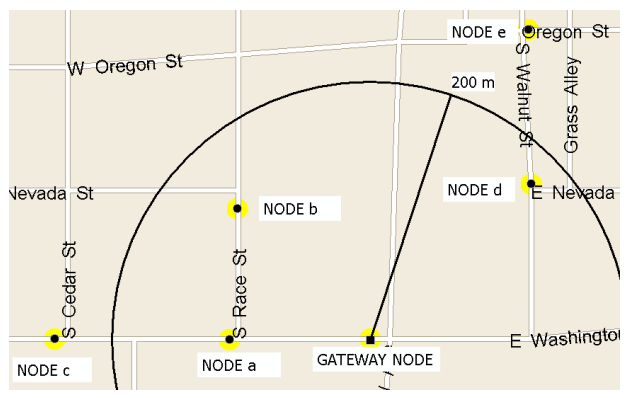

(a) Geographic locations of mesh nodes.

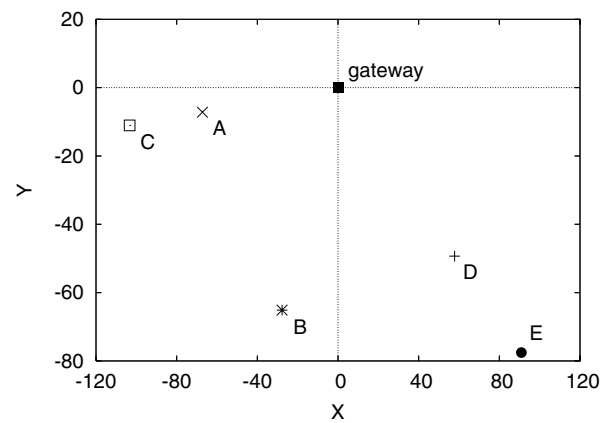

(b) Locations in signal strength based coordinate system.

Figure 5: Locations of mesh nodes in the geographic map and the virtual coordinate system.

observation, they proposed the Link RED algorithm that controls the link-layer packet dropping probability in order to keep the TCP window size around the optimal value.

Note that all the aforementioned approaches make direct efforts to minimize the interference by tuning physical layer parameters. In contrast, we measure the signal strengths received between nodes, and use them to infer the interference level between nodes that are apart from each other by more than the transmission range. The results are then used to coordinate frame transmission among connections with the least inter-flow interference. From the perspective of detecting (rather than mitigating) interference, the work by Nadeem et al. [26] comes closest to ours. They use the geographic locations of wireless nodes to obtain the path loss model and to predict the interference. When stations transmit frames, they include various transmission parameters such as their geographic location, antenna gain, and transmission power in order to enable an overhearing station within their transmission range to predict whether a new transmission may adversely affect any ongoing transmission.

\section{PERFORMANCE EVALUATION}

\subsection{Empirical Evaluation w.r.t. Accuracy in Constructing the Coordinate System}

We first evaluate the performance of the coordinate system with respect to the accuracy in determining the node coordinates. Recall that under some cases the coordinates of two-hop neighbor nodes are derived with approximations (Eq. (7)). One important step in validating the coordinate system is then to verify whether or not the distance between two nodes in the coordinate system is truly representative of their received signal strength. The study is performed with real-life measurements of received signal strengths between nodes on the Champaign-Urbana Community Wireless Network (CUWiN). Figure 5(a) shows the geographic map of a gateway node and its 5 neighboring mesh nodes. The gateway node has a transmission range of approximately $200 \mathrm{~m}$, and has three onehop neighbor nodes: $\mathbf{a}, \mathbf{b}$, and $\mathbf{d}$. Among these nodes, the gateway node, $\mathbf{a}$, and $\mathbf{b}$ can directly communicate with each other, while $\mathbf{d}$ can only directly communicate with the gateway node and $\mathbf{b}$. The signal strength matrix $\mathbf{S}$ (of dimension 3 ) is obtained as

$$
\mathbf{S}=\left(\begin{array}{ccc}
0 & 75 & 71 \\
60 & 0 & 70 \\
71 & 70 & 0
\end{array}\right)
$$

where $s_{i j}$ is (- RSS) in $\mathrm{dBm}$. Figure 5(b) gives the 2-dimensional coordinate system obtained with the mean vector of [48.6, 43.3,
Table 1: Parameters used in ns-2 simulations.

\begin{tabular}{|c|c||c|c|}
\hline Propagation & Two-ray & Antenna height & $1.5 \mathrm{~m}$ \\
\hline Tx power & $8.58 \mathrm{dBm}$ & SNR thresh & $10 \mathrm{~dB}$ \\
\hline Rx thresh & $-64.37 \mathrm{dBm}$ & CS thresh & $-78.07 \mathrm{dBm}$ \\
\hline Data rate & $11 \mathrm{Mb} / \mathrm{s}$ & $N$ in Algorithm 1 & 10 \\
\hline
\end{tabular}

47.0] in Eq. (1) and the scale factor $\alpha$ of 0.704 in Eq. (2). The discrepancy between the measured signal strengths and the computed Euclidean distances in Figure 5(b) is $2.6 \mathrm{dBm}$ on average. This is due to the fact that $s_{12} \neq s_{21}$ (as a result of asymmetric path loss). If we replace both $s_{12}$ and $s_{21}$ with its average of 67.5, the error becomes as small as $0.15 \mathrm{dBm}$.

Node $\mathbf{d}$ is the neighbor of the gateway node, but is out of the transmission range of $\mathbf{a}$. In this case, the coordinate is obtained as the solution to Eq. (4). The coordinates of the two two-hop neighbors of the gateway node, $\mathbf{c}$ and $\mathbf{e}$, are computed by Eq. (7) with the use of the signal strength measurement at their corresponding onehop neighbors ( $\beta=4$ is used in the calculation). In Figure 5(b), the Euclidean distance between $\mathbf{b}$ and $\mathbf{c}$ is 92.92 , and the actual RSS of $\mathbf{c}$ at $\mathbf{b}$ is $-85 \mathrm{dBm}$. The discrepancy is $7.92 \mathrm{dBm}$, and is a result of the fact that a two-hop neighbor is assumed to lie in the same direction in the coordinate system as the one-hop neighbor node. As the node density increases, more signal strength measurements are available, and the the error discrepancy is expected to be alleviated. This implies the distance in the proposed coordinate system can accurately represent the signal strength in reasonably populated networks. We will study the impact of inaccurate locations of two-hop neighbors on the throughput performance below (in Figures 9).

\subsection{Simulation in Various Topologies}

To evaluate the performance of CoTx and compare it against IEEE 802.11 DCF [16], we have carried out simulation in $n s-2$ in a variety of network scenarios. Table 1 gives the default parameter setting used in the simulation study. With the parameter setting the transmission range is approximately $100 \mathrm{~m}$ and the carrier sense range is $220 \mathrm{~m}$. Because we use the two-ray propagation model in ns-2, the signal strength between two nodes is simply a function of their distance. However, we observe a variation of $2.3842 \mathrm{~dB}$ in the signal strength in real-life measurements and have incorporated an additive Gaussian noise term in the two-ray propagation model to improve simulation fidelity.

Performance evaluation in the star topology. We first consider the star topology and show how temporal-spatial diversity 


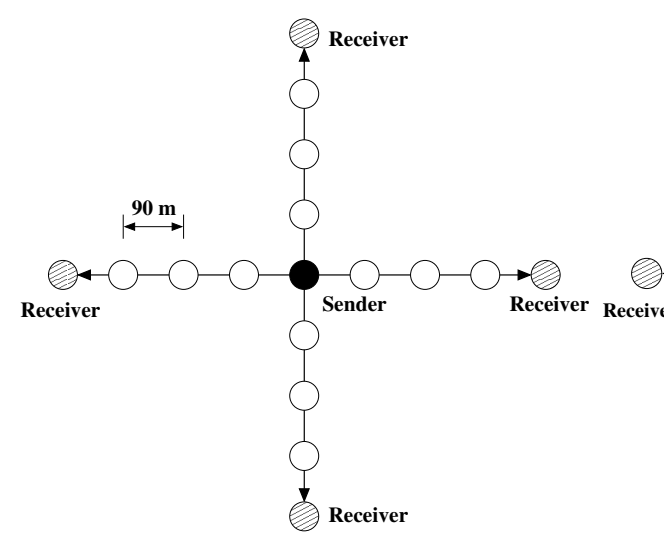

(a) 4 branches.

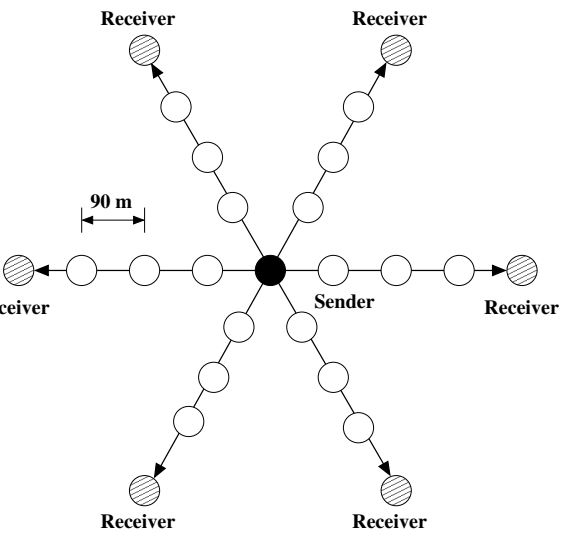

(b) 6 branches.

Figure 6: Star topologies with multihop wireless paths. The transmission range is $100 \mathrm{~m}$ and the interference range is $220 \mathrm{~m}$.

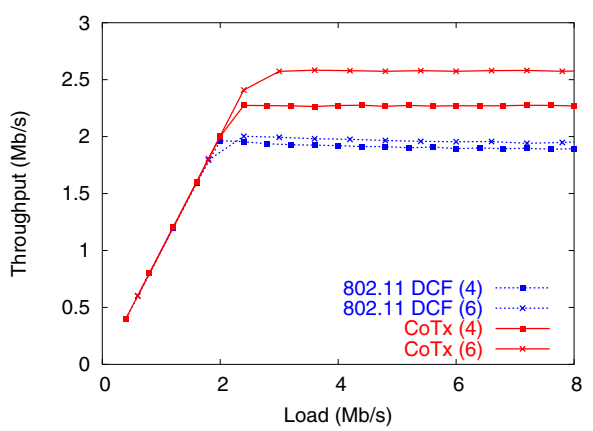

(a) Traffic load.

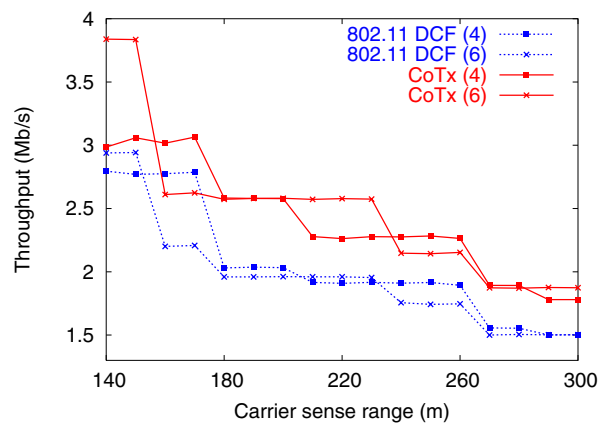

(b) Carrier sense range.

Figure 7: Throughput performance with respect to the offered traffic load and the carrier sense range in the star topologies. The number in the parenthesis indicates the number of branches in the topology.

can be exploited to improve the network throughput. Figure 6(a) and (b) give the star topology with 4 and 6 branches, respectively. The distance between adjacent nodes on a wireless path is $90 \mathrm{~m}$. Recall that in Section 2 the simple topology of 2 multi-hop paths illustrates how intra-/inter-flow interference degrades the throughput performance in multihop wireless networks.

First, we vary the offered traffic load by changing the frame sending rates of CBR connections from the gateway node to the receivers. Figure 7(a) gives the aggregate throughput of receivers with respect to the offered load at the gateway node. Under light traffic load, IEEE 802.11 DCF and CoTx give the same performance. When the traffic is higher than $2 \mathrm{Mb} / \mathrm{s}$, the network is saturated, and CoTx achieves better throughput. The performance improvement is, respectively, 20.1 and $31.8 \%$ for the 4 and 6-branch star topology. Furthermore, while the bandwidth obtained 802.11 DCF in the 6-branch topology does not differ from that in the 4branch topology, the bandwidth under CoTx increases by $300 \mathrm{~Kb} / \mathrm{s}$ in Figure 7(a). Under the two-ray propagation model, the SIR at a two-hop neighbor of the gateway node is computed by $\left(4 \sin \frac{\theta}{2}\right)^{4}$, where $\theta$ is the angle between adjacent paths, (i.e., $\frac{\pi}{2}$ and $\frac{\pi}{3}$ for each case in Figure 6). Because the computed SIR's are greater than the threshold of $10 \mathrm{~dB}$, CoTx determines that all the paths do not interfere with one another and performs bulk transmission with consecutive 4 and 6 packets, respectively. This implies that CoTx is capable of discovering and exploiting the temporal-spatial diversity.
Next, we vary the carrier sense range by changing its threshold while keeping the transmission power constant. We observe that as the carrier sense range increases, the throughput for both methods decreases in Figure 7(b). This means that this carrier sense threshold is set unnecessarily small, and reduces the number of concurrent transmissions on the network. Even in the case, CoTx still attains better throughput than IEEE 802.11 DCF for all the carrier sense threshold values tested. The performance improvement is as much as $27.2 \%$ for the 4-branch star topology and $31.7 \%$ for the 6-branch star topology.

Performance evaluation in grid and random topologies. We carry out simulation in a larger network of size $1080 \mathrm{~m}$ $\mathrm{x} 1080 \mathrm{~m}$, where the gateway node is located at the center, and the other 168 nodes are placed either regularly at the grid as in Figure 8(a) and randomly as in Figure 8(b). A total of 10 client nodes are randomly selected and CBR connections are established from the gateway node to these selected client nodes. In this set of simulation, we also consider CoTx with one-hop approximation, i.e., the coordinates of two-hop nodes are assumed to lie (rather than at its actual geographical location) on the straight line that connects from the location of the gateway node to the location of its corresponding one-hop node, and is predicted with Eq. (7).

We vary the transmission range (and accordingly the carrier sense range), the carrier sense range (for a fixed transmission range), and the offered traffic load. As shown in Figures 9, CoTx gives higher throughput than IEEE 802.11 DCF in most cases. Several obser- 


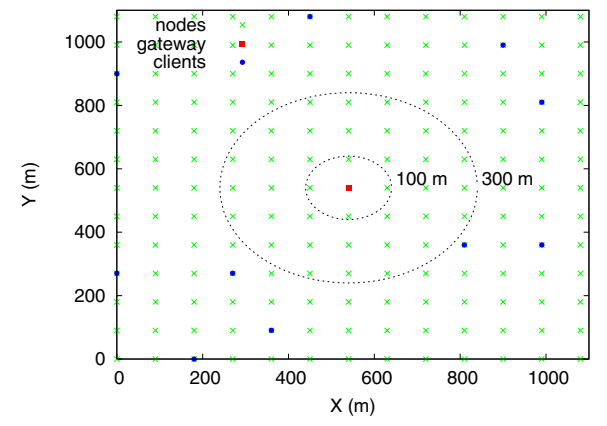

(a) Grid topology.

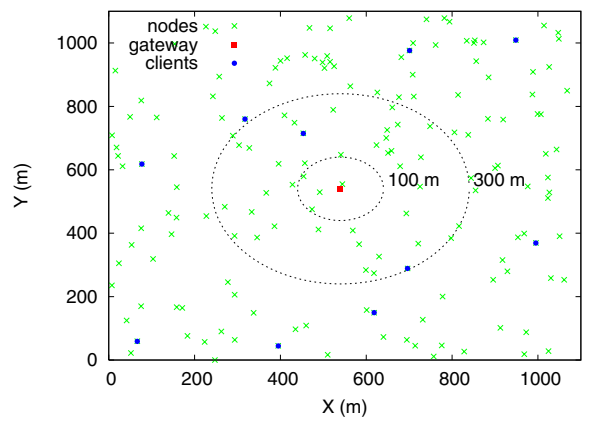

(b) Random topology.

Figure 8: Grid and random topologies with one gateway node and 168 mesh nodes.

vations are in order: First, the throughput of CoTx with one-hop approximation is only slightly worse than that obtained with exact locations. This implies that the location of the first-hop node is the most dominant factor in inferring the signal strength between two-hop nodes, because it essentially determines the direction of the path. This also implies it is possible to use CoTx in a sparse network without significant loss in the throughput performance.

Second, whether or not temporal-spatial diversity can be fully exploited depends highly on the nodal distribution within the transmission range of the gateway node. If there are a number of nodes evenly distributed within the transmission range of the gateway node, the chance for bulk transmission with a larger number of frame increases. In the random topology shown in Figure 8(b), most nodes that are within a distance of $100 \mathrm{~m}$ are located in the lower left part of the topology, and the throughput attained by CoTx is almost the same as that by IEEE 802.11 DCF as shown in Figure 9(b).

Last, both the transmission range and the carrier sense range affect spatial reuse and hence should be properly tuned to increase spatial reuse. If the transmit power is increased to the degree that multiple paths exist near the gateway node, CoTx can have more chances to select one-hop neighbors with least interference. However, because the increase of the transmit power causes that of the interference level to its neighbors, the transmit power for CoTx should be carefully set to maximize the overall spatial reuse. As part of our future work, we will leverage the work in [24, 35] to select the best transmit power and carrier sense threshold to further improve the performance of CoTx.

Performance evaluation w.r.t. various parameter settings. CoTx has two tunable parameters: the SIR threshold and the loss exponential factor. The SIR threshold is used to determine whether two nodes are within the interference range in Eq. (8). If the threshold is set to a small value, more paths will be determined to be interference-free and the bulk transmission will be packed with more frames. This leads to higher throughput, but also increases inter-flow interference. Figure 10(a) gives the throughput performance when the SIR threshold varies from 0 to $30 \mathrm{~dB}$. Note that the SNR threshold in ns-2 is still set to be $10 \mathrm{~dB}$. Since the number of paths in our grid and random topologies is geographically restricted, small threshold values do not degrade the throughput performance. However, large threshold values prevent path diversity from being fully exploited, and the attainable throughput under CoTx eventually reduces to that under IEEE 802.11 DCF.

The loss exponential factor is used to estimate the coordinates of two-hop nodes in Eq. (7). Figure 10(b) gives the throughput performance with respect to different values of the factor. This parameter does not affect the performance significantly. As long as a same loss factor is exclusively used to infer the interference levels among different paths, its relative effect on decision making is not significant.

\section{CONCLUSION}

In this paper, we have investigated how to mitigate the inter/intra-flow interferences in multiple-hop wireless mesh networks by exploiting temporal-spatial diversity of concurrent transmissions that exist among a number of wireless links. We proposed an coordinate system that represents the signal strength received between nodes as the distance, from which the inter-flow interference among transmitters that are out of each other's transmission range can be accurately inferred. With the use of the node coordinates in the system, we identify connections that potentially do not interfere with each other, and coordinate their frame transmission by enabling gateway nodes to transmit in bulk. Through extensive simulation with real-life measurement traces, we showed that the proposed CoTx scheme alleviates as much as $35 \%$ of throughput degradation caused by interference.

We have identified several future research avenues. First, CoTx can, in principle, be applied not only to gateway nodes but also to relay nodes in wireless mesh networks. When a relay node identifies itself as a performance bottleneck, it can select paths with the least interference and coordinate frame transmission in the same fashion gateway nodes do. Second, as mentioned in Section 6.2, although we do not make any assumption on the transmit power and the carrier sense threshold, they are crucial to achieving better throughput performance in multiple-hop wireless networks. As complimentary research to CoTx, we will attempt to determine the best combination of the transmit power and the carrier sense threshold [18]. The network throughput is expected to improve with the combination of CoTx and better tuning of transmit power/carrier sense threshold. Third, the coordinate system for inferring the interference between nodes can be established with a different characteristic metric such as packet delivery rate [29]. Finally, we are in the process of populating the Champaign-Urbana Community Wireless Network with more nodes (from 30 to 60) and implementing CoTx in the gateway nodes, and will empirically evaluate the performance of CoTx. 


\section{REFERENCES}

[1] Bay area wireless user group. http://www.bawug.org/.

[2] Champaign-Urbana community wireless network. http://www.cuwireless.net/.

[3] MIT roofnet. http://pdos.csail.mit.edu/roofnet/doku.php.

[4] Seattle wireless. http://www.seattlewireless.net/.

[5] SFLan. http://www.sflan.org/.

[6] Southampton open wireless network. http://www.sown.org.uk/.

[7] A. Adya, P. Bahl, J. Padhye, A. Wolman, and L. Zhou. A multi-radio unification protocol for IEEE 802.11 wireless networks. In IEEE BroadNets, July 2004.

[8] D. Aguayo, J. Bicket, S. Biswas, G. Judd, and R. Morris. Link-level measurements from an $802.11 \mathrm{~b}$ mesh network. In Proc. of ACM SIGCOMM, September 2004.

[9] D. Allen. Hidden terminal problems in wireless LAN's. 802.11 Working Group paper 802.11/93-xx.

[10] D. Berger, Z. Ye, P. Sinha, S. Krishnamurthy, M. Faloutsos, and S. K. Tripathi. TCP-friendly medium access control for ad-hoc wireless networks: alleviating self-contention. In Proc. of IEEE MASS, October 2004.

[11] J. Bicket, D. Aguayo, S. Biswas, and R. Morris. Architecture and evaluation of an $802.11 \mathrm{~b}$ mesh network. In Proc. of ACM Mobicom, September 2005.

[12] D. S. J. De Couto, D. Aguayo, J. C. Bicket, and R. Morris. A high-throughput path metric for multi-hop wireless routing. In ACM Mobicom, 2003.

[13] R. Draves, J. Padhye, and B. Zill. Comparison of routing metrics for static multi-hop wireless networks. In Proc. of ACM SIGCOMM, September 2004.

[14] R. Draves, J. Padhye, and B. Zill. Routing in multi-radio, multi-hop wireless mesh networks. In Proc. of ACM MOBICOM, 2004.

[15] Z. Fu, H. Luo, P. Zerfos, S. Lu, L. Zhang, and M. Gerla. The impact of multihop wireless channel on TCP performance. IEEE Trans. on Mobile Computing, 4(2):209-221, March/April 2005.

[16] IEEE. Wireless LAN Media Access Control (MAC) and Physical Layer (PHY) Specification. IEEE Standard 802.11, 1999.

[17] IEEE Computer Society. Part 11: Wireless LAN Medium Access (MAC) and Physical Layer (PHY) Specifications, Amendment 8: Medium Access Cntorl (MAC) Quality of Service Enhancement. IEEE Standard 802.11e, 2005.

[18] T.-S. Kim, H. Lim, and J. C. Hou. Improving spatial reuse through tuning transmit power, carrier sense threshold, and data rate in multihop wireless networks. In Proc. of ACM MobiCom, 2006.

[19] K. Langendoen and N. Reijers. Distributed localization in wireless sensor networks: a quantitative comparison. Elsevier Computer Networks, 43:499-518, 2003.

[20] H. Lim, J. C. Hou, and C.-H. Choi. Constructing Internet coordinate system based on delay measurement. IEEE/ACM Transactions on Networking, 13(3):513-525, 2005.
[21] R. T. B. Ma, S. C. M. Lee, J. C. S. Lui, and D. K. Y. Yau. A game theoretic approach to provide incentive and service differentiation in P2P networks. In Proc. ACM

SIGMETRICS, New York, NY, June 2004.

[22] Microsoft Networking Research Group. Self-organizing neighborhood wireless mesh networks. http://research.microsoft.com/mesh/.

[23] J. Monks, V. Bharghavan, and W.-M. Hwu. A power controlled multiple access protocol for wireless packet networks. In Proc. of IEEE INFOCOM, 2001.

[24] A. Muqattash and M. Krunz. Power controlled dual channel (PCDC) medium access protocol for wireless ad hoc networks. In Proc. of IEEE INFOCOM, 2003.

[25] A. Muqattash and M. Krunz. A single-channel solution for transmission power control in wireless ad hoc networks. In Proc. of ACM Mobihoc, 2004.

[26] T. Nadeem, L. Ji, A. Agrawala, and J. Agre. Location enhancement to IEEE 802.11 DCF. In Proc. of IEEE INFOCOM, March 2005.

[27] B. Noble and J. W. Daniel. Applied Linear Algebra. Prentice Hall, 1988.

[28] Nortel Networks. The business case for wireless mesh networks. http://www.nortelnetworks.com/corporate/events/2003d /wmn_eseminar/collateral/wmn_eseminar.pdf, December 2003.

[29] J. Padhye, S. Agarwal, V. N. Padmanabhan, L. Qiu, A. Rao, and B. Zill. Estimation of link interference in static multi-hop wireless networks. In Proc. of Internet Measurement Conference, 2005.

[30] B. Raman and K. Chebrolu. Design and evaluation of a new MAC protocol for long-distance 802.11 mesh networks. In Proc. of ACM MOBICOM, 2005.

[31] A. Raniwala and T. Chiueh. Architecture and algorithms for an IEEE 802.11-based multi-channel wireless mesh network. In Proc. of IEEE INFOCOM, 2005.

[32] K. Sanzgiri, I. D. Chakeres, and E. M. Belding-Royer. Determining intra-flow contention along multihop paths in wireless networks. In Proc. of Broadnets Wireless Networking Symposium, October 2004.

[33] S. Xu and T. Saadawi. Does the IEEE 802.11 MAC protocol work well in multihop wireless adhoc networks? IEEE Communications Magazine, 39(6):130-137, 2001.

[34] X. Yang and N. Vaidya. On the physical carrier sense in wireless ad-hoc networks. In Proc. of IEEE INFOCOM, 2005.

[35] J. Zhu, X. Guo, L. Yang, and W. S. Conner. Leveraging spatial reuse in 802.11 mesh networks with enhanced physical carrier sensing. In Proc. of IEEE ICC, 2004.

[36] J. Zhu, S. Roy, X. Guo, and W. S. Conner. Maximizing aggregate throughput in 802.11 mesh networks with physical carrier sensring and two-radio multichannel clustering. In Proc. of NFS-RPI Workshop on Pervasive Computing and Networking, 2004. 
Grid Topology

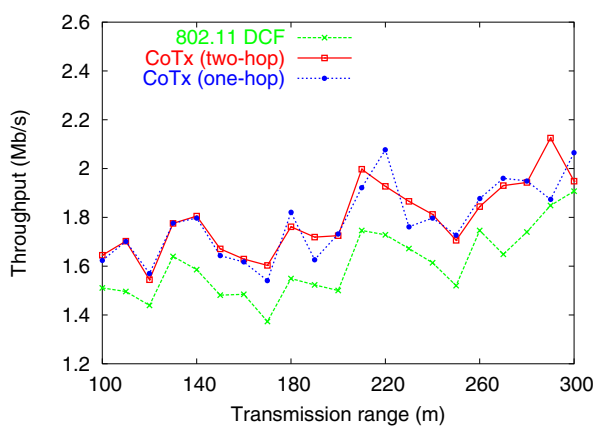

(a) Transmission range (load $=5 \mathrm{Mb} / \mathrm{s})$.

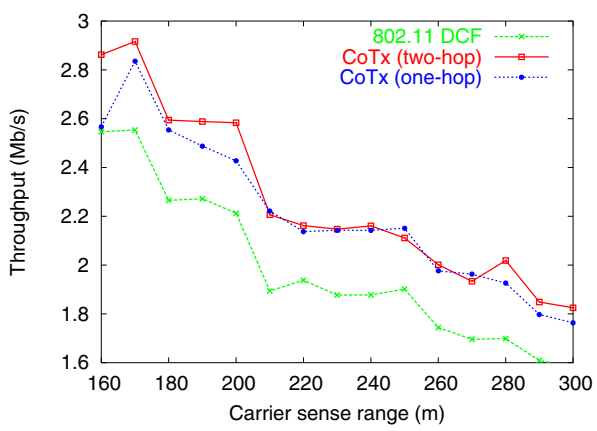

(c) Carrier sense range (load $=5 \mathrm{Mb} / \mathrm{s}$ and tx range $=150 \mathrm{~m}$ ).

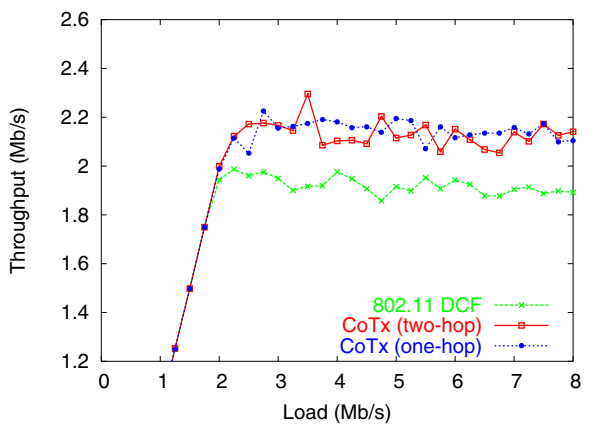

(e) Traffic load (tx range $=150 \mathrm{~m}$ and cs range $=220 \mathrm{~m}$ ).
Random Topology

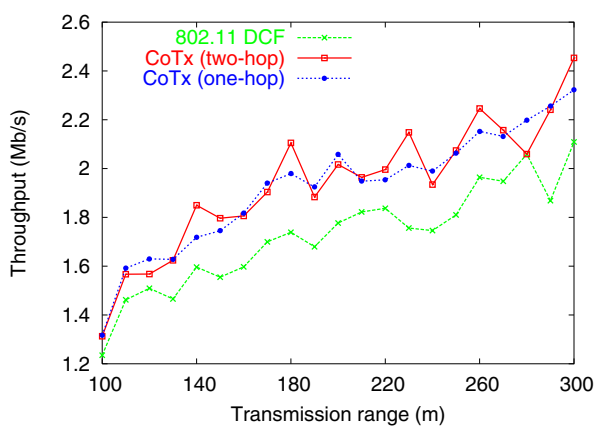

(b) Transmission range (load $=5 \mathrm{Mb} / \mathrm{s})$.

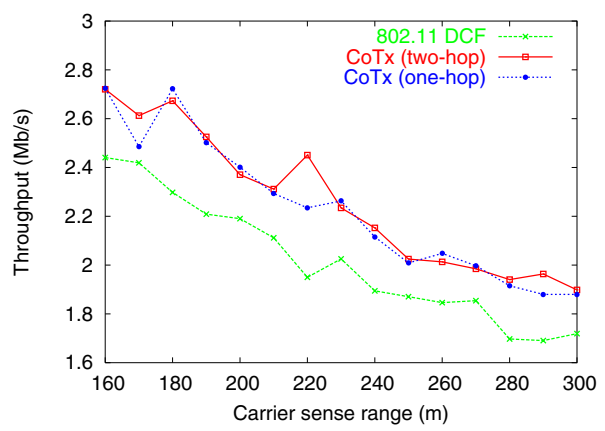

(d) Carrier sense range (load $=5 \mathrm{Mb} / \mathrm{s}$ and tx range $=150 \mathrm{~m}$ ).

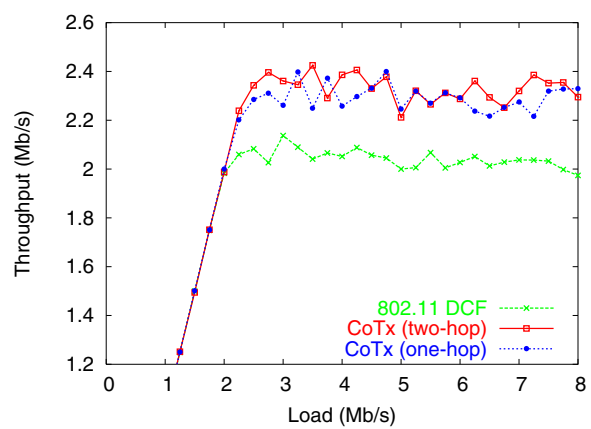

(f) Traffic load (tx range $=150 \mathrm{~m}$ and cs range $=220 \mathrm{~m}$ ).

Figure 9: Throughput performance with respect to the transmission range, the carrier sense range, and the traffic load in the grid and the random topologies.

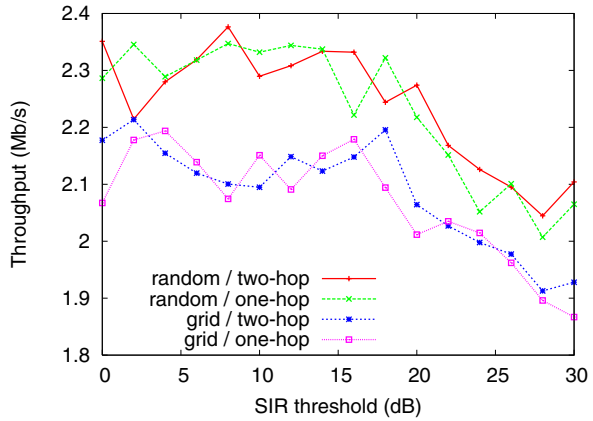

(a) SIR threshold.

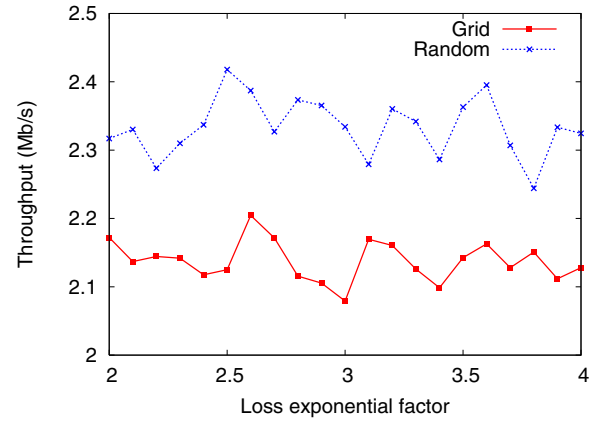

(b) Loss exponential factor.

Figure 10: Throughput performance with respect to two parameters used in CoTx: the SIR threshold and the loss exponential factor. 